EPJ Web of Conferences 28, 09013 (2012)

DOI: 10.1051/epjconf/20122809013

(C) Owned by the authors, published by EDP Sciences, 2012

\title{
Exotic Physics Searches at CMS
}

\author{
Bryan Dahmes on behalf of the CMS Collaboration \\ University of Minnesota, School of Physics and Astronomy, 116 Church Street S.E., Minneapolis, MN 55455
}

\begin{abstract}
We summarize the results of several searches for evidence of new physics phenomena using protonproton collisions at $\sqrt{s}=7 \mathrm{TeV}$ delivered by the Large Hadron Collider at CERN and recorded by the CMS detector in 2011.
\end{abstract}

\section{Introduction}

The Standard Model (SM) has proven itself over many decades to be in very good agreement with experimental results. Despite this success, we realize that the SM is an incomplete theory and we therefore anticipate that the data delivered by the Large Hadron Collider (LHC) at CERN will contain evidence for new phenomena outside the current framework.

In this Note we summarize several searches for exotic new physics processes not currently explained by the Standard Model ${ }^{1}$. These searches were conducted using $\sqrt{s}=7 \mathrm{TeV} p p$ collision data delivered by the LHC and collected by the CMS detector [1] in 2011 .

\section{Searches for New Heavy Charged Gauge Bosons}

Various extensions to the Standard Model predict that new heavy gauge bosons may be found with TeV-scale masses. One such example is the $W^{\prime}$, which may be considered a heavy analogue to the SM W boson and possess the same left-handed fermionic couplings. CMS searches for this boson using $1.1 \mathrm{fb}^{-1}$ of collision data via its leptonic decay mode $W^{\prime} \rightarrow \ell v$, reconstructing final states containing an electron or muon and a neutrino [2]. By examining the transverse mass distribution of the lepton and neutrino, where the neutrino presence in the detector is inferred from the missing transverse energy in the event $\left(E_{T}^{\text {miss }}\right)$, we find the data are in good agreement with SM expectations. Assuming SM-like couplings, we exclude $W^{\prime}$ bosons with mass below $2.27 \mathrm{TeV}$ at the $95 \%$ confidence level (C.L.).

Due to the large expected mass of the $W^{\prime}$, the decay $W^{\prime} \rightarrow W Z$ is also allowed, and if the leptonic decays of the $W^{\prime}$ are suppressed the decay rate to the $W Z$ final state could be relatively enhanced. We also consider that certain versions of Technicolor, a strongly interacting gauge theory which allows for the dynamical breakdown of electroweak

\footnotetext{
1 This note is not intended to summarize all searches for new physics phenomena conducted by CMS. Discussions of CMS searches for the Higgs boson, Supersymmetry, and exotic resonances can be found in other proceedings for this conference.
}

symmetry, describe techni-particles (e.g. $\rho_{\mathrm{TC}}$ ) that may decay to the $W Z$ final state with narrow resonant peaks. Searching in the $W Z$ final state with $1.1 \mathrm{fb}^{-1}$ of data, where both the $W$ and $Z$ decay leptonically, we exclude SM-like $W^{\prime}$ bosons with mass below $784 \mathrm{GeV}$ [3]. We also compare our results in the $W Z$ final states to predictions from Technicolor models and present $95 \%$ C.L. exclusion for $\rho_{\mathrm{TC}}$ and $\pi_{\mathrm{TC}}$ in Figure 1.

Finally, we consider the possibility that the heavy W boson originates from an extension to the SM that includes a right-handed $\mathrm{SU}(2)$ symmetry group and search for the decay $W_{R} \rightarrow \ell N_{R}$, where the heavy neutrino $N_{R}$ is the right-handed analog of the SM neutrino $v_{L}$. Assuming righthanded couplings for the new heavy gauge boson, using $0.2 \mathrm{fb}^{-1}$ we exclude in the two-dimensional $\left(M_{W_{R}}, M_{N_{R}}\right)$ space that extends to $M_{W_{R}}=1.7 \mathrm{TeV}$ [4].

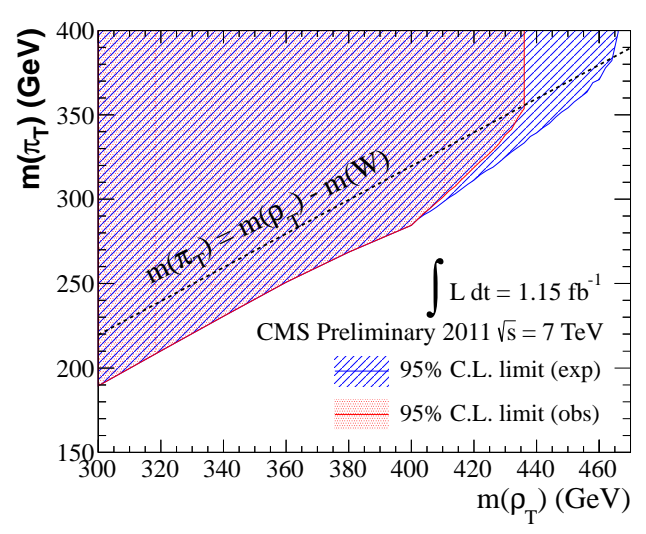

Fig. 1. Two-dimensional exclusion limit for Technicolor in the $W Z$ final state as a function of the $\rho_{\mathrm{TC}}$ and $\pi_{\mathrm{TC}}$ masses.

\section{Searches for Fourth Generation and Excited Quarks}

Precision electroweak measurements imply that the Standard Model can only accommodate three fermion generations. However, recently there has been renewed interest in extending the SM to include a fourth generation of 
fermions. Assuming a fourth generation, the indirect bounds on the mass of the Higgs boson could be relaxed, and a heavy fourth generation neutrino could be considered as a dark matter candidate. An additional generation of quarks may also possess enough intrinsic matter and anti-matter asymmetry to contribute to the baryon asymmetry of the Universe.

Given this motivation, CMS searches for fourth generation $b^{\prime}$ and $t^{\prime}$ quarks, produced singly or in pairs, considering multiple final states. We search for pair-production of $b^{\prime}$ quarks via the decay chain $b^{\prime} \bar{b}^{\prime} \rightarrow t W^{-} \bar{t} W^{+}$and consider final states with three leptons $(\ell=e, \mu)$ or same-sign dileptons [5]. We also search for $t^{\prime} \bar{t}^{\prime} \rightarrow b W^{+} \bar{b} W^{-}$, and consider final states where one [6] or both [7] of the $W$ bosons decays leptonically. Standard Model expectations, which are dominated by $t \bar{t}$ production, are in good agreement with the results obtained using $1.1 \mathrm{fb}^{-1}$ of data. As a result, we exclude heavy top-like (bottom-like) quarks with masses below $450 \mathrm{GeV}(495 \mathrm{GeV})$ at the $95 \%$ confidence level.

We additionally consider mixing between the third and fourth generations and search for fourth generation quarks produced singly $\left(t^{\prime} b\right.$ or $\left.b^{\prime} t\right)$ or in pairs as a function of the four-generation CKM matrix parameter $A$, where $V_{t b}=$ $V_{t^{\prime} b^{\prime}}=\sqrt{A}$ and $V_{t^{\prime} b}=V_{t b^{\prime}}=\sqrt{1-A}$ [8]. This inclusive search requires at least one of the $W$ bosons in the decay chain to decay to a muon and neutrino, and events are categorized according to the number of $b$-tagged hadronic jets and reconstructed $W \rightarrow q \bar{q}^{\prime}$ candidates. We find no evidence for fourth generation quarks in this search. Assuming degenerate $t^{\prime}$ and $b^{\prime}$ masses, we summarize the $95 \%$ C.L. exclusion limits on fourth generation quark masses as a function of $A$ in Figure 2.

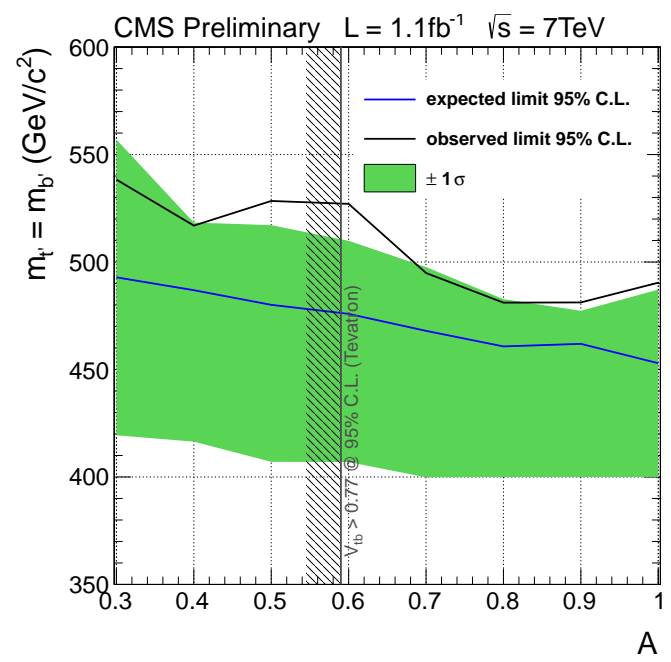

Fig. 2. Expected and observed exclusion limits for $M_{t^{\prime}}=M_{b^{\prime}}$ as a function of the four-generation CKM parameter A as described in the text. The parameter values below the solid black line are excluded at $95 \%$ C.L.

A search for vector-like quarks $(T)$ with charge $|q|=\frac{2}{3}$ is also performed by CMS [9] using $1.1 \mathrm{fb}^{-1}$ of collision data. We search for $p p \rightarrow T T$ production, followed by $T \rightarrow t Z \rightarrow b W Z$. Motivated by this decay chain, we search for events with at least two jets and at least three leptons, requiring that two of the leptons be consistent with $Z \rightarrow \ell \ell$ decay. Based on these requirements, backgrounds are dominated by events with real $Z$ bosons. We find no excess beyond SM expectations, and conclude that $M_{T}>475 \mathrm{GeV}$ at the $95 \%$ confidence level.

\section{Search for Extra Dimensions}

The great disparity between the Planck scale $\left(M_{\mathrm{Pl}} \sim 10^{19} \mathrm{GeV}\right)$ and the electroweak scale $\left(M_{\mathrm{EW}} \sim 10^{3} \mathrm{GeV}\right)$ is a great mystery of the Standard Model. The model proposed by Arkani-Hamed, Dimopoulos, and Dvali (ADD) includes a framework for addressing this hierarchy problem. Taking the fundamental scale to be of the same order as the electroweak scale $\left(M_{D} \sim M_{\mathrm{EW}}\right)$, the observed weakness of gravitational interactions (equivalently, the large Planck mass) would be a consequence of the universe having compactified extra dimensions. In the ADD model, the SM gauge interactions are constrained to the familiar 3 (space) +1 (time) dimensional subspace while gravity is allowed to propagate through the entire multidimensional space. Taking these hypothesized extra dimensions into account, the Planck scale is related to the fundamental scale by $M_{\mathrm{Pl}}^{2} \sim$ $M_{D}^{n+2} r^{n}$, where $r$ and $n$ are the size and number of extra dimensions, respectively. If $M_{D}$ is at the TeV scale, it would be possible to produce gravitons at the LHC.

Gravitons $(G)$ could be produced directly at the LHC via the processes $g g \rightarrow q G, q \bar{q} \rightarrow g G, q g \rightarrow q G$, and $q \bar{q} \rightarrow g G$. As the graviton escapes detection, we must search for evidence for extra dimensions in final states that include large $E_{T}^{\text {miss }}$. CMS performs two searches of this nature, as we search for final states involving a real graviton balanced by a photon [10] or hadronic jet $(j)$ [11]. In each case, events are rejected if additional jets or isolated tracks are found with significant transverse energy, and additional requirements suppress beam backgrounds, cosmic ray signatures, and instrumental noise that may mimic the desired final state. We present the leading jet transverse momentum spectrum for the $j+E_{T}^{\text {miss }}$ channel in Figure 3. Both the $\gamma+E_{T}^{\text {miss }}$ and $j+E_{T}^{\text {miss }}$ searches find no evidence for direct graviton production using $1.1 \mathrm{fb}^{-1}$ of data, and set limits on the fundamental mass scale $M_{D}$ that depend on the number of extra dimensions considered.

Virtual graviton exchange is also possible at the LHC, which could lead to enhanced non-resonant dimuon production, which we consider in [12]. Using $1.1 \mathrm{fb}^{-1}$ of data, we find results are consistent with SM expectations. We consequently set $95 \%$ C.L. lower limits on the fundamental mass scale depend on the number of extra dimensions and additional theoretical conventions used to describe virtual graviton production.

\subsection{Search for Microscopic Black Holes}

One of the exciting potential consequences of theoretical models with extra spatial dimensions and lowscale quantum gravity is the copious production of microscopic black holes in particle collisions at the LHC. We analyze events with large total transverse energy, searching for multiple high energy jets, leptons, and photons which are expected from the decay of black holes produced at LHC energies [13]. Backgrounds from Standard Model processes, which are 


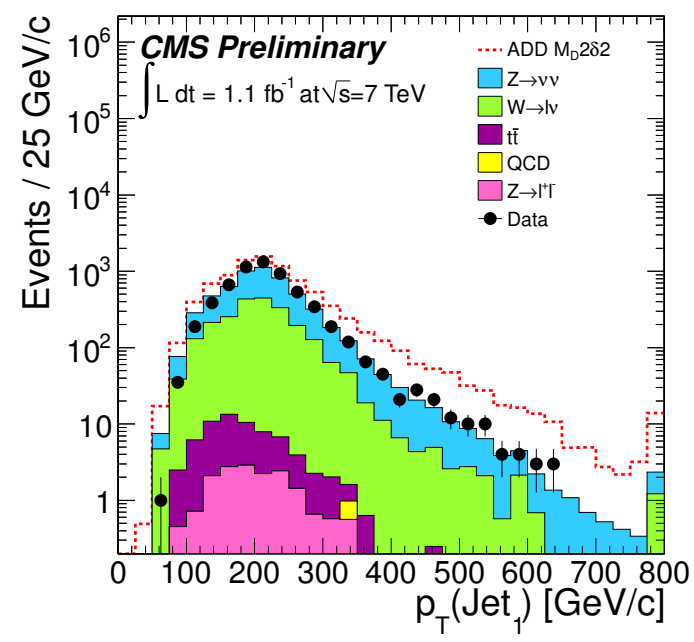

Fig. 3. Transverse momentum distribution for the leading jet in the jet $+E_{T}^{\text {miss }}$ search for extra dimensions. The leading jet is required to have $p_{T}>100 \mathrm{GeV}$ and be reconstructed within the acceptance of the CMS tracker.

dominated by QCD multijet production, are in good agreement with the results found in data. We therefore set limits on the minimum black hole mass that depend on the number of ADD extra dimensions and the fundamental mass scale $M_{D}$.

\section{Search for R-Parity Violation in Multilepton Events}

One common feature of many Supersymmetric (SUSY) extensions to the Standard Model is conservation of " $\mathrm{R}$ parity". Conserving this quantity forces Supersymmetric particles to be produced in pairs and ensures the lightest SUSY particle (LSP) is stable and thus a good dark matter candidate. As the LSP escapes detection, many SUSY searches require $E_{T}^{\text {miss }}$ in the final state to account for the loss of the LSP.

If we instead consider models with R-parity violating interactions, the LSP loses stability and consequently decays to SM particles that could be observed in the detector. As $E_{T}^{\text {miss }}$ loses significance as a search quantity, we instead search for deviations from Standard Model expectations in events with significant total transverse energy [14]. Using $2.1 \mathrm{fb}^{-1}$ of data collected in 2011, we consider 52 exclusive channels, categorized according to the number of leptons $(\ell=e, \mu)$ and same-flavor $\ell^{+} \ell^{-}$pairs in the event, the number of reconstructed $\tau$ leptons in the event, whether or not a $Z \rightarrow \ell \ell$ decay is reconstructed, and the total transverse energy in the event. Several channels are dominated by SM processes and serve as control channels, while other channels expect negligible SM background contributions. Datadriven methods are used to estimate background levels, including an estimate of asymmetric photon conversions using $Z \rightarrow \mu \mu \gamma \rightarrow 4 \mu$ events. We see good overall agreement with background expectations across all channels, and set limits on coupling strengths for R-parity violating SUSY interactions. One such limit is shown in Figure 4.

In two channels with high total transverse energy, four leptons, and at least one same-flavor $\ell^{+} \ell^{-}$pair, we expect low backgrounds from SM processes but find a total of two events, finding one event each in channel (with or without a $Z$ candidate). While interesting, additional luminosity is required before we can access the significance of the result.

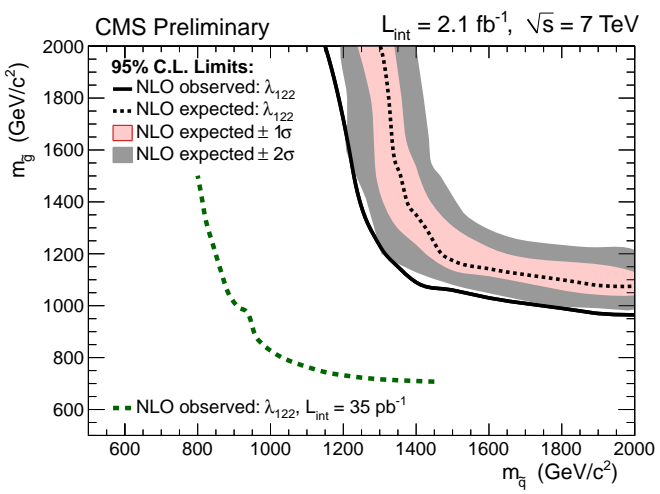

Fig. 4. 95\% C.L. limits for R-Parity violating coupling $\lambda_{122}=$ $\lambda_{e \mu \mu}$ as a function of the squark and gluino masses for a SUSY topology described in [14]. The observed limits, along with limits expected in the absence of signal are shown, in addition to the uncertainty in the expectation. Masses to the left of the curves are excluded.

\section{Search for Heavy Long-Lived Particles}

\subsection{Charged Particles}

Heavy quasi-stable charged particles appear in various extensions to the Standard Model. Heavy long-lived particles with a hadronic character, such as gluinos or stop squarks, will hadronize in flight and form meta-stable bound states with quarks and gluons. If the lifetime of these so-called "R-hadrons" produced at the LHC exceeds a few nanoseconds, the particles will travel over distances that are comparable or larger than the size of the CMS detector. Depending on particle lifetime and interaction with the detector material, CMS applies two complimentary techniques when searching for heavy long-lived charged particles.

The first approach assumes that a significant fraction of the R-hadrons will have a velocity smaller than $0.9 c$. As a result, these particles will be directly observable through the distinctive signature of a high momentum particle with an anomalously large rate of ionization energy loss in the CMS tracker and an anomalously long time-of-flight as measured by the CMS Muon system [15]. We search for Rhadrons traversing the entire detector using both the tracker and muon systems, and additionally search using only the tracker information to account for the possibility that the particle may become neutral before reaching the muon detectors. Using $1.1 \mathrm{fb}^{-1}$ of data collected in 2011 , searches for anomalous charged tracks allow us to exclude the production of gluinos, hadronizing into stable R-gluonballs with $10 \%$ (50\%) probability, with masses below 899 (839) GeV at $95 \%$ confidence level. The analogous limit on stop production is $620 \mathrm{GeV}$.

We also perform a search for low velocity $(v<0.4 c)$ Rhadrons, where the energy loss is great enough that a significant fraction of the produced particles come to rest in 
the CMS detector volume [16]. These "stopped" R-hadrons will then decay to hadronic jets at some later time depending on the unknown particle lifetime. The decay of these particles will occur out-of-time with respect to LHC $p p$ collisions, so our online (trigger) selection for stopped particles places a veto on the beam presence. In a dataset with a peak instantaneous luminosity of $1.3 \times 10^{33} \mathrm{~cm}^{-2} \mathrm{~s}^{-1}$, an integrated luminosity of up to $886 \mathrm{pb}^{-1}$ lifetime, and a search interval corresponding to 168 hours of trigger live time, no significant excess above background (mostly from instrumental noise) was observed. Limits at the $95 \%$ confidence level on R-hadron pair production extend over 13 orders of magnitude of gluino lifetime.

\subsection{Neutral Particles}

CMS also searches for long-lived neutral particles that are present in several exotic physics models. In one such search, we consider pair production of long-lived neutral particles (e.g. $H \rightarrow \chi \chi$ ), each of which subsequently decays to a dielectron or dimuon pair [17]. As the massive neutral particles travel some distance in the detector before decaying, the $\chi \rightarrow \ell^{+} \ell^{-}$decay vertex will be displaced with respect to the $p p$ collision vertex. Using $1.1 \mathrm{fb}^{-1}$ of collision data, we select $e^{+} e^{-}$and $\mu^{+} \mu^{-}$combinations that have large transverse decay lengths relative to the decay length uncertainty. A single event survives the $e^{+} e^{-}$selection, consistent with expectations from $Z \rightarrow e e$ decay. No events survive the muon selection. In Figure 5 we present the $95 \%$ C.L. exclusion limits as a function of the decay length of the heavy neutral particle.
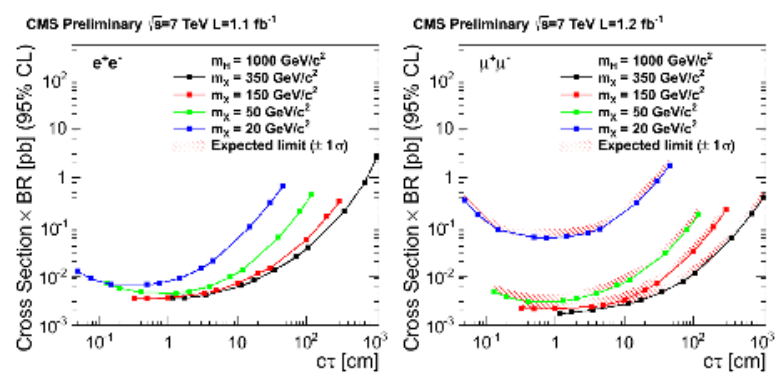

Fig. 5. 95\% confidence level upper limits on the cross section and branching fraction for the assumed $H \rightarrow \chi \chi$ decay, with $\chi \rightarrow e e$ (left) and $\chi \rightarrow \mu \mu$ (right), assuming a Higgs mass of $1000 \mathrm{GeV}$. The expected limit band for the electron channel is too small to be visible.

In some cases, the long-lived neutral particle may decay exclusively to other neutral particles (one example is the decay of a neutralino to a photon and a gravatino). In these cases, we can exploit the large amount of tracker material in CMS to estimate the particle lifetime [18]. We search for photons that convert in the detector to a dielectron pair. As the four-momentum of the $\gamma \rightarrow e^{+} e^{-}$pair points along the same direction as the parent photon, the tracks of the electrons can be precisely reconstructed and used to calculate the photon trajectory and, in particular, the impact parameter of the photon with respect to the interaction point. Examining $2.1 \mathrm{fb}^{-1}$ of collision data for a photon with significant impact parameter in association with missing transverse energy (due to the escaping gravatino), we find the data is in good agreement with expectations from SM processes and determine the upper limits for the cross section for pair-production of neutralinos, each of which decays into one photon and invisible particles, as a function of neutralino lifetime.

\section{Conclusion}

In this Note we summarize several searches for exotic new physics processes not currently explained by the Standard Model. In each search, the data are in good agreement with expectations from Standard Model processes. We proceed to set $95 \%$ C.L. limits on the parameters and particle masses relevant for various new physics scenarios.

\section{Acknowledgments}

The author wishes to thank the organizers of the Hadron Collider Physics Symposium for the rich and interesting physics program and the excellent conference location.

\section{References}

1. The CMS Collaboration, JINST 3 (2008) S08004.

2. The CMS Collaboration, CMS Physics Analysis Summary EXO-11-024 (2011).

3. The CMS Collaboration, CMS Physics Analysis Summary EXO-11-041 (2011).

4. The CMS Collaboration, CMS Physics Analysis Summary EXO-11-002 (2011).

5. The CMS Collaboration, CMS Physics Analysis Summary EXO-11-036 (2011).

6. The CMS Collaboration, CMS Physics Analysis Summary EXO-11-050 (2011).

7. The CMS Collaboration, CMS Physics Analysis Summary EXO-11-051 (2011).

8. The CMS Collaboration, CMS Physics Analysis Summary EXO-11-054 (2011).

9. The CMS Collaboration, Phys. Rev. Lett. 107 (2011) 271802.

10. The CMS Collaboration, CMS Physics Analysis Summary EXO-11-058 (2011).

11. The CMS Collaboration, CMS Physics Analysis Summary EXO-11-059 (2011).

12. The CMS Collaboration, CMS Physics Analysis Summary EXO-11-039 (2011).

13. The CMS Collaboration, CMS Physics Analysis Summary EXO-11-071 (2011).

14. The CMS Collaboration, CMS Physics Analysis Summary EXO-11-045 (2011).

15. The CMS Collaboration, CMS Physics Analysis Summary EXO-11-022 (2011).

16. The CMS Collaboration, CMS Physics Analysis Summary EXO-11-020 (2011).

17. The CMS Collaboration, CMS Physics Analysis Summary EXO-11-004 (2011).

18. The CMS Collaboration, CMS Physics Analysis Summary EXO-11-067 (2011). 